\title{
Identification of a novel antisense noncoding RNA, ALID, transcribed from the putative imprinting control region of marsupial IGF2R
}

\author{
Shunsuke Suzuki ${ }^{1,2}$ (D) Geoffrey Shaw ${ }^{3}$ and Marilyn B. Renfree ${ }^{3 *}$ (1)
}

\begin{abstract}
Background: Genomic imprinting leads to maternal expression of IGF2R in both mouse and opossum. In mouse, the antisense long noncoding (Inc) RNA Airn, which is paternally expressed from the differentially methylated region $(D M R)$ in the second intron of Igf2r, is required to silence the paternal Igf2r. In opossum, however, intriguingly, the DMR was reported to be in a different downstream intron (intron 11) and there was no antisense IncRNA detected in previous analyses. Therefore, clarifying the imprinting mechanism of marsupial IGF2R is of great relevance for understanding the origin and evolution of genomic imprinting in the IGF2R locus. Thus, the antisense IncRNA associated with the marsupial DMR can be considered as the 'missing link'. In this study, we identified a novel antisense IncRNA, ALID, after detailed analysis of the IGF2R locus in an Australian marsupial, the tammar wallaby, Macropus eugenii, and compared it to that of the grey short-tailed opossum, Monodelphis domestica.

Results: Tammar IGF2R showed maternal expression and had a maternally methylated CpG island (CGI) in intron 12 as well as a promoter CGI without differential methylation, but none in the second intron. Re-analysis of the IGF2R of opossum detected the CGI in intron 12, not intron 11, as previously reported, confirming that the DMR in intron 12 is conserved between these marsupials and so is the putative imprinting control region of marsupial IGF2R. ALID is paternally expressed from the middle of the DMR and is approximately $650 \mathrm{bp}$ long with a single exon structure that is extremely short compared to Airn. Hence, the IncRNA transcriptional overlap of the IGF2R promoter, which is essential for the lgf2r silencing in the mouse, is likely absent in tammar. This suggests that fundamental differences in the IncRNA-based silencing mechanisms evolved in eutherian and marsupial IGF2R and may reflect the lack of differential methylation in the promoter CGI of marsupial IGF2R.
\end{abstract}

Conclusions: Our study thus provides the best candidate factor for establishing paternal silencing of marsupial IGF2R without transcriptional overlap, which is distinct from the Igf2r silencing mechanism of Airn, but which may be analogous to the mode of action for the flanking S/c22a2 and S/c22a3 gene silencing in the mouse placenta.

Keywords: Genomic imprinting, IGF2R, IncRNA, Marsupials

\footnotetext{
*Correspondence: m.renfree@unimelb.edu.au

${ }^{3}$ School of BioSciences, The University of Melbourne, Victoria 3010,

Australia

Full list of author information is available at the end of the article
}

(c) The Author(s) 2018. This article is distributed under the terms of the Creative Commons Attribution 4.0 International License (http://creativecommons.org/licenses/by/4.0/), which permits unrestricted use, distribution, and reproduction in any medium, provided you give appropriate credit to the original author(s) and the source, provide a link to the Creative Commons license, and indicate if changes were made. The Creative Commons Public Domain Dedication waiver (http://creativecommons.org/ publicdomain/zero/1.0/) applies to the data made available in this article, unless otherwise stated. 


\section{Background}

Genomic imprinting is an epigenetic mechanism which regulates parent-of-origin-dependent expression of imprinted genes. In higher vertebrates, it has been observed only in mammals and is limited to viviparous mammalian groups, the eutherians and marsupials [1-6] and appears to be absent in the egg-lying monotremes [7]. While more than 100 imprinted genes have been identified in eutherians so far, it has become clear that only small subset of them are also imprinted in marsupials [8-17]. Most imprinted genes are highly expressed in the placenta, and functions of imprinted genes are often associated with placental development and growth, foetal and postnatal development and growth, control of maternal behaviour related to postnatal care and also lactation $[3-5,18]$. Therefore, the evolution of genomic imprinting might be correlated with the evolution of mammalian viviparity. The study the divergent evolutionary pathways taken by therian mammals of imprinting mechanism would contribute to understanding how mammals have acquired complex epigenomic regulation because genomic imprinting has been an excellent model revealing many epigenetic mechanisms to control gene expression.

The mouse Igf2r locus on chromosome 17 is one of the best characterised imprinted domains which includes the maternally expressed $I g f 2 r$, Slc22a2 and Slc22a3 genes and the paternally expressed lncRNA gene Airn [19, 20]. The Airn lncRNA is an established example of a cissilencing lncRNA essential for the paternal silencing of Igf $2 r$, Slc22a2 and Slc22a3 [21-24]. Airn is transcribed from the maternally methylated DMR located in the $I g f 2 r$ intron 2 in antisense orientation against to $\operatorname{Igf} 2 r[25,26]$. Airn transcription overlaps the Igf $2 r$ promoter but not the $S l c 22 a 2$ or Slc22a3 promoters. Airn transcriptional overlap of the Igf $2 r$ promoter, not its lncRNA products, is required for paternal silencing of $I g f 2 r$, interfering with RNA polymerase II recruitment to the Igf $2 r$ promoter [27]. On the other hand, paternal silencing of Slc22a3 in the placenta depends on the Airn lncRNA products recruiting the H3K9 histone methyltransferase G9a to the Slc22a3 promoter [28]. Thus, Airn has two different modes of action to silence paternal Igf2r and Slc22a3.

In humans, adult tissues lack IGF2R imprinted expression $[29,30]$, but it is found in foetal tissues, placenta, cultured amniotic cells, lymphoblastoid cells and Wilms' tumours where it is polymorphic [31-34]. The human IGF2R intronic CpG island has promoter activity, and the human orthologue of Airn is present at least in Wilms' tumours [35]. IGF2R shows imprinted expression also in sheep and dogs, but not in pigs [36-38]. Although the imprinted expression pattern of $I G F 2 R$ is not identical in these eutherian species, the intron $2 \mathrm{CpG}$ islands are maternally methylated equally.

In marsupials, there is maternal expression of IGF2R in the opossum, Monodelphis domestica [39]. However, interestingly, Das et al. [40] found a maternally methylated DMR in opossum IGF2R intron 11 (we argue that it is intron 12, see discussion) not intron 2 and there was no antisense IncRNA found around the opossum IGF2R DMR. Given the presence of marsupial IGF2R DMR at a different genomic location from that of the mouse, studying the imprinting control mechanism in the marsupial $I G F 2 R$ locus would be of great importance to understand the evolution of genomic imprinting in mammals. Hence, the antisense lncRNA associated with the marsupial DMR can be considered as the 'missing link' to investigate imprinting mechanism of marsupial IGF2R unless there is an as yet unidentified lncRNA-independent mechanism that regulates marsupial IGF2R imprinting.

In this study, we demonstrate that tammar IGF2R is also maternally expressed and that the CGI located in tammar IGF2R intron 12 is a maternally methylated DMR. Furthermore, we identify a novel paternally expressed antisense lncRNA, ALID (Antisense LncRNA in the IGF2R DMR), which is transcribed from the middle of the tammar IGF2R DMR. Because of the extremely short 650 bp length of ALID compared to Airn, lncRNA transcriptional overlap of the IGF2R promoter, which is essential for the $I g f 2 r$ silencing in the case of mouse, is likely absent in tammar. This suggests that there is fundamental difference in the lncRNA-based silencing mechanisms between eutherian and marsupial IGF2R. Our study thus provides the most likely candidate factor for establishing paternal silencing of marsupial IGF2R without transcriptional overlap, which is distinct from the Igf $2 r$ silencing mechanism of Airn, but may be analogous to mode of action for the flanking Slc22a3 silencing in the mouse placenta.

\section{Results \\ Maternal expression of tammar IGF2R}

We first searched for polymorphism in the $3^{\prime}$ untranslated region (UTR) of tammar IGF2R to distinguish paternal and maternal alleles. Only single nucleotide polymorphism (SNP) site was found at the very end of the last exon with A/G sequence variation (Fig. 1a). This SNP enabled us to perform restriction fragment length polymorphism (RFLP) analysis by the presence or absence of the CviAII restriction recognition site (Fig. 1a). After CviAII digestion for the PCR products amplified from genomic DNA and cDNA of bilaminar and trilaminar regions of the yolk sac placentae and of fibroblastlike primary cell cultures as well as maternal DNA, the intensity of cut and uncut bands in gel electrophoresis 


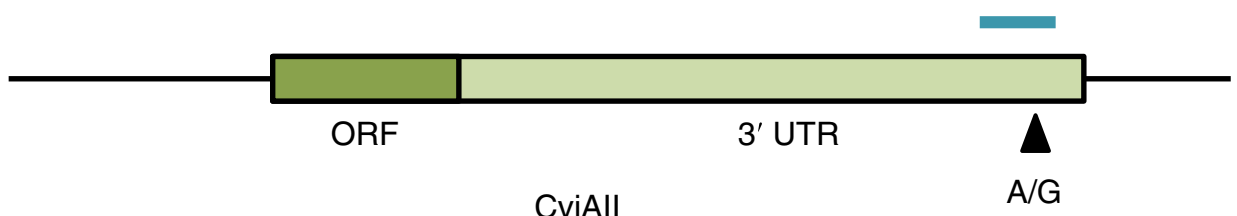

G Allele

(CATG)

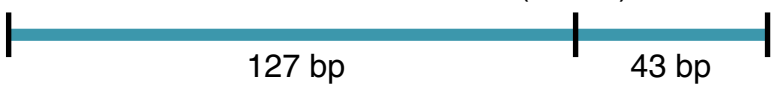

A Allele
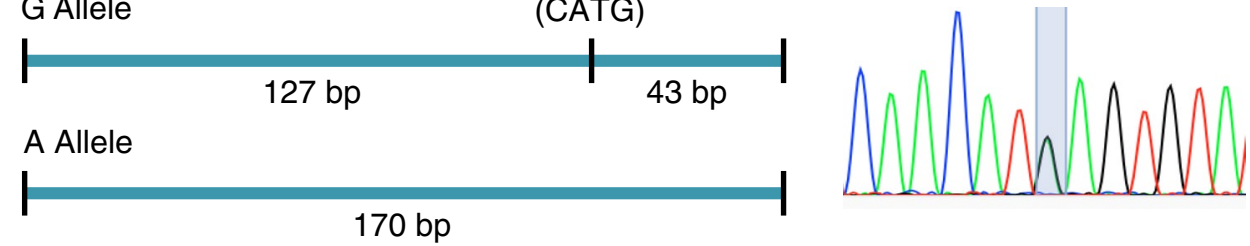

b

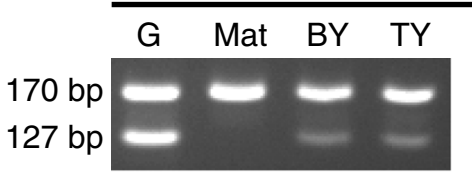

$\# 1$

CviAll

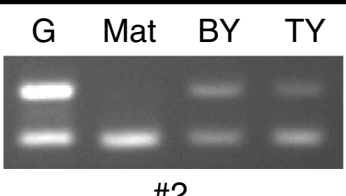

\#2

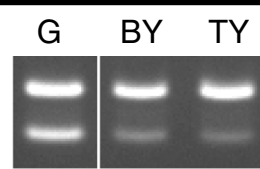

\#3

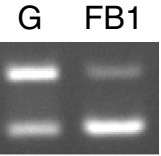

\#4

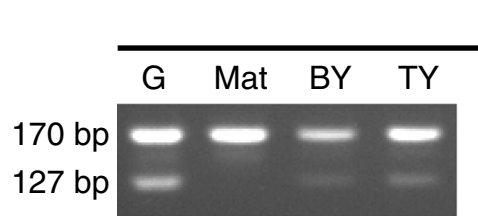

\#5

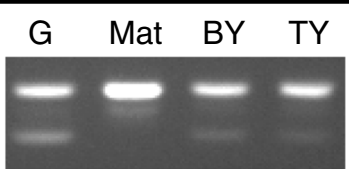

\#6

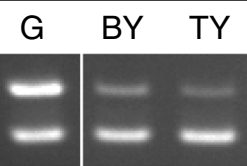

\#7

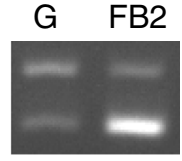

\#8

c

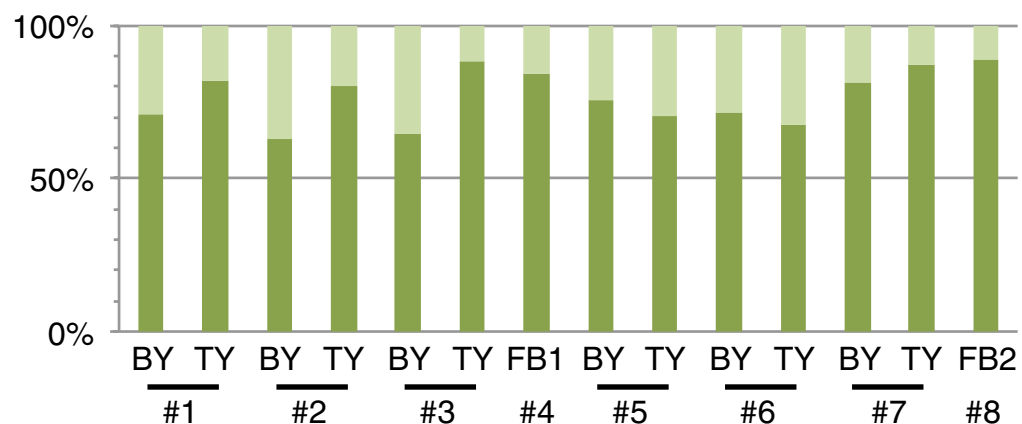

Fig. 1 Imprinting analysis of tammar IGF2R. a Single nucleotide polymorphism in tammar /GF2R. The dark and light green parts in the box represent ORF and 3' UTR of the last exon of tammar IGF2R, respectively. The black arrowhead and light blue line show the position of polymorphic site and the region of PCR amplification that were used for allelic expression analysis by RFLP analysis, respectively. The 170 bp PCR product contains a recognition sequence of CviAll restriction enzyme for $G$ allele but not for $A$ allele, enabling RFLP analysis. The sequencing data of a heterozygous sample show double peak of $A$ and $G$ at the polymorphic site. $\mathbf{b}$ Allelic expression pattern of tammar IGF2R. The intensity of 170 and 127 bp bands shows expression level from A allele and G allele, respectively. G genomic DNA, Mat maternal genotype, BY yolk sac placenta (bilaminar region), TY yolk sac placenta (trilaminar region), FB fibroblast cell line, \#; individual number. c Allelic expression ratio of tammar IGF2R. The dark and light green bars represent expression ratio of active and inactive alleles, respectively. Each ratio was calculated under the normalisation using genomic DNA data as $50 \%$ 
was quantified. Expression of tammar IGF2R was clearly biased to either the $A$ or $G$ alleles that produce uncut and cut bands, respectively (Fig. 1b, c). In the 4 individuals, $\# 1,3,5,6$, the A allele predominantly expressed $I G F 2 R$ mRNA while in the other 4 cases, $\# 2,4,7,8$, reciprocally biased expression was observed (Fig. 1b). Of these, the 4 informative cases for maternal genotype, \#1, 2, 5, 6 , showed that maternal allele was predominantly transcribed. These data demonstrated that tammar IGF2R is also a maternally expressed imprinted gene as in mouse and opossum.

\section{DNA methylation analysis of $\mathrm{CpG}$ islands in the promoter and intron 12 of tammar IGF2R}

Given the imprinting of tammar IGF2R, we next analysed DNA methylation to investigate the possible imprinting mechanism of the IGF2R locus in marsupials. One CGI was located over the putative promoter region of tammar IGF2R, while the other CGI was found in intron 12 at an orthologous position to the opossum intron 11 CGI which forms a DMR (Fig. 2a). Bisulphite sequencing revealed that most $\mathrm{CPG}$ sites were unmethylated in almost the entire region of the promoter CGI, suggesting that differential epigenetic modification other than DNA methylation at the promoter region, such as histone modification, regulates maternal expression of tammar IGF2R (Fig. 2b). In contrast, clear differential methylation was observed in the intron 12 CGI (Fig. 2c). The two informative cases having a SNP inside the amplified region enabled us to determine that the methylated allele was maternally transmitted. Thus, it was demonstrated that the intron $12 \mathrm{CGI}$ is a maternally methylated DMR and the putative imprinting control region of marsupial IGF2R.

\section{Detection and determination of the structure of ALID IncRNA}

To compare the regulatory mechanisms of IGF2R imprinting between eutherians and marsupials, we examined whether there is any antisense lncRNA which is transcribed from intron 12 DMR similar to Airn of the mouse Igf $2 r$ locus. To selectively reverse transcribe antisense transcripts, strand-specific RT-PCR was performed using a reverse transcription primer designed at the upstream region of the DMR in intron 12 in sense orientation against IGF2R (Fig. 3a). The results indicated the presence of any antisense transcripts nearby the DMR, although expression level seemed not to be high in the yolk sac placenta. Hence we next carried out $5^{\prime}$ and $3^{\prime}$ RACE (rapid amplification of cDNA ends) to determine the full-length structure of this antisense transcript (Fig. 3b, c). Sequencing the nested $5^{\prime}$ RACE product, it appeared that the transcription start site (TSS) of the antisense transcript was located in the middle of the DMR, suggesting that this antisense transcript is the marsupial equivalent to murine Airn (Fig. 3d). Combining the data of $3^{\prime}$ RACE, it was revealed that this antisense transcript is approximately 650 bp long with a single exon structure and no obvious protein-coding potential. A typical polyadenylation signal was located before the polyA tail, suggesting the result of $3^{\prime}$ RACE was not an artefact (Fig. 3d). Thus, we named this antisense lncRNA ALID.

\section{Expression and imprinting analyses of ALID}

We confirmed expression of ALID in a range of tissues from developing young by both conventional PCR and strand-specific reverse transcription followed by qPCR. $A L I D$ was ubiquitously expressed in various stages of tammar pouch young although there was some degree of difference in expression levels between tissues (Fig. 4a, b). The imprinting status of ALID was determined by direct sequencing of strand-specific RT-PCR products that contain a SNP site. In contrast to the data of genomic DNA PCR products in which double peaks at SNP site have almost the same signal strength, the data of RT-PCR products clearly showed that $A L I D$ is predominantly expressed from either one of the two alleles (Fig. 4c). Remarkably, in the data of individual \#2, the expressed allele was opposite to the data of \#1 and \#4, showing that the monoallelic expression observed in these data was not consequence of simple allelic difference but reflected the parental origin. DNA methylation analysis of the nearby genomic region including these SNP sites showed that the expressed alleles were unmethylated alleles (data

\footnotetext{
(See figure on next page.)

Fig. 2 DNA methylation analysis in tammar IGF2R. a Location of CpG islands in opossum and tammar IGF2R. The graphs show the density of CpG sites in genomic sequences including opossum and tammar IGF2R, respectively. The yellow highlighted peaks in the graphs indicate the promoter and intron $12 \mathrm{CpG}$ islands in opossum IGF2R and the orthologous CpG islands detected in tammar IGF2R genomic sequence. b DNA methylation analysis of promoter CpG island in tammar IGF2R. The red bars indicate each CpG site in $3 \mathrm{~kb}$ genomic sequence including the tammar IGF2R promoter $\mathrm{CpG}$ island. The green lines represent the regions analysed by bisulphite sequencing. Open circle; unmethylated CpG site, filled circle; methylated CpG site. c DNA methylation analysis of intron $12 \mathrm{CpG}$ island in tammar IGF2R. The red line in \#4 data represents the location of a single nucleotide polymorphism. Direct sequencing of PCR products amplified from the mother's genomic DNA clarified maternal allele-specific DNA methylation
} 
a Gray Short-tailed Opossum IGF2R

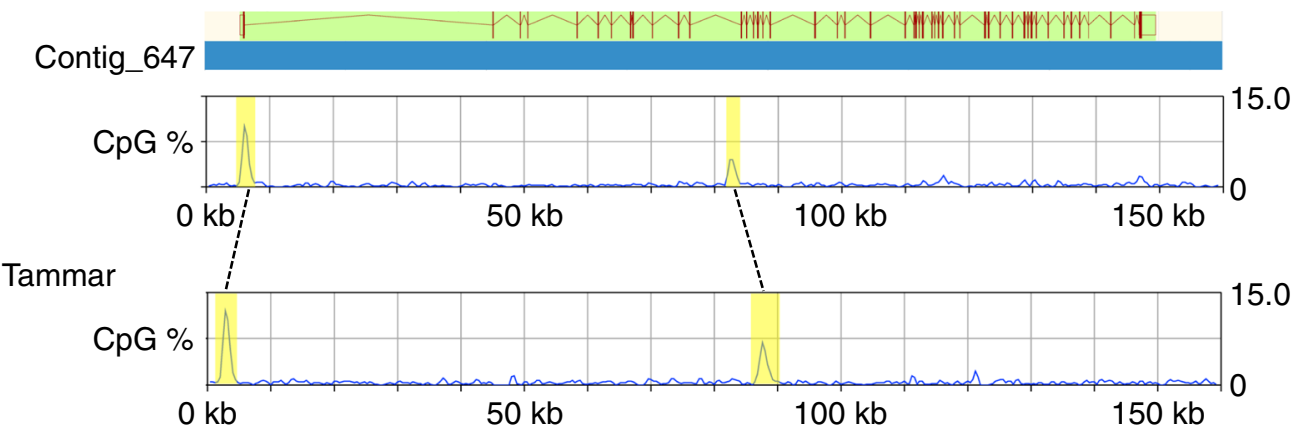

b

Tammar IGF2R

Promoter CpG Island
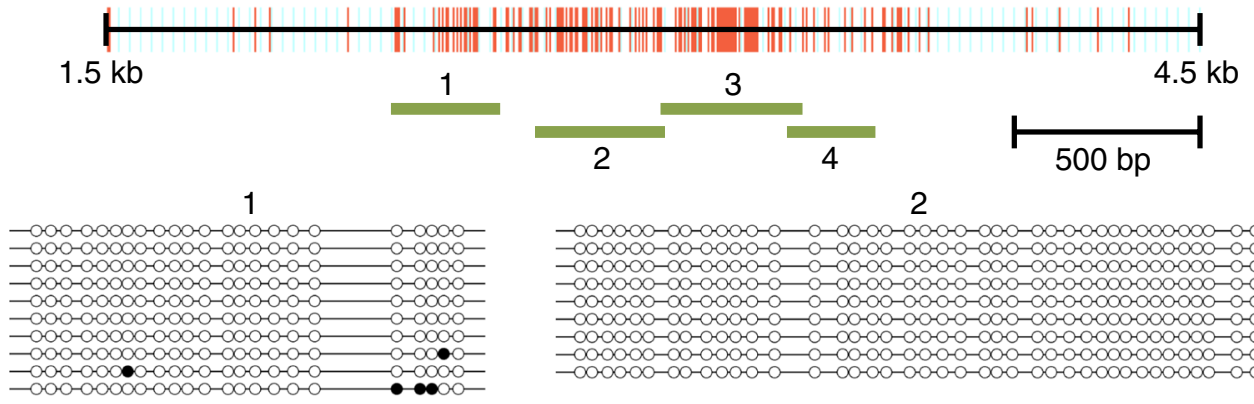

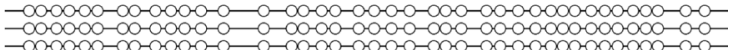

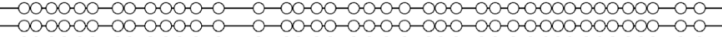

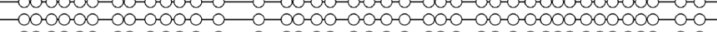

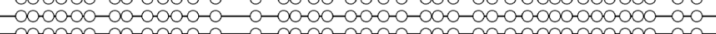

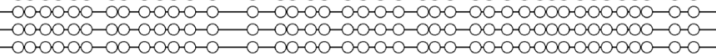

3

4
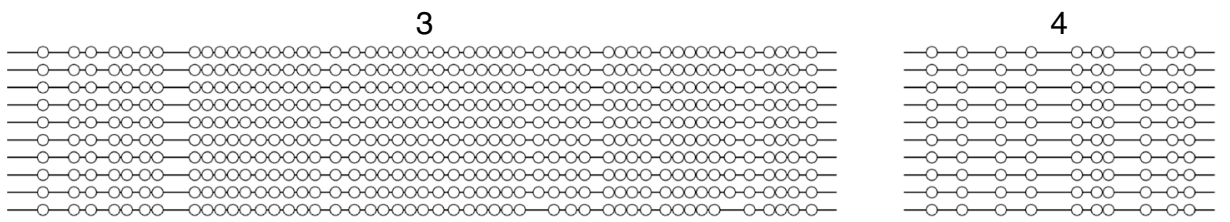

c

Tammar IGF2R

Intron $12 \mathrm{CpG}$ Island

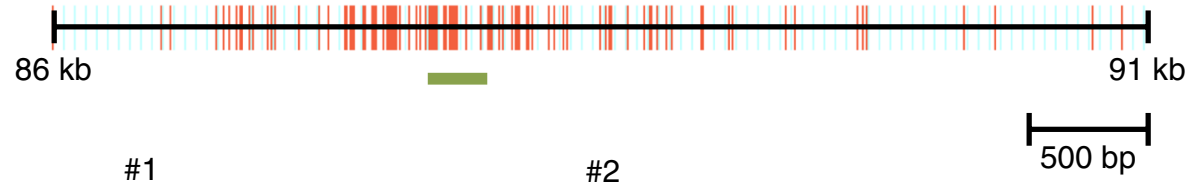

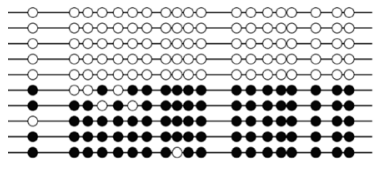

\#3
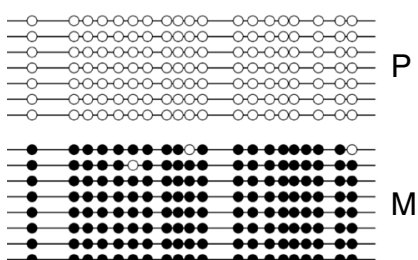

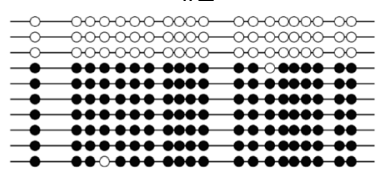

\#4

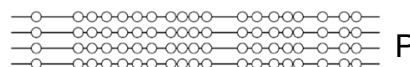

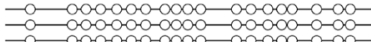

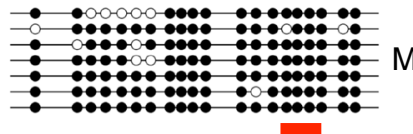

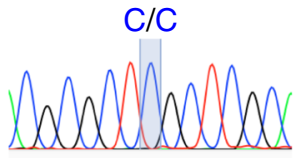

\#4 Mother Genomic Seq.

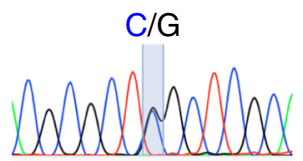

\#4 Genomic Seq. 
a

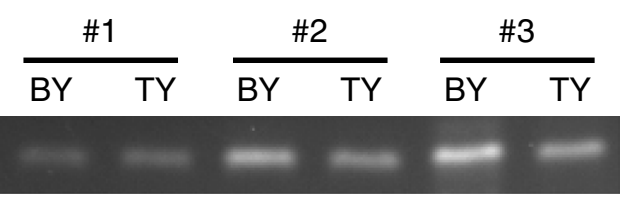

b

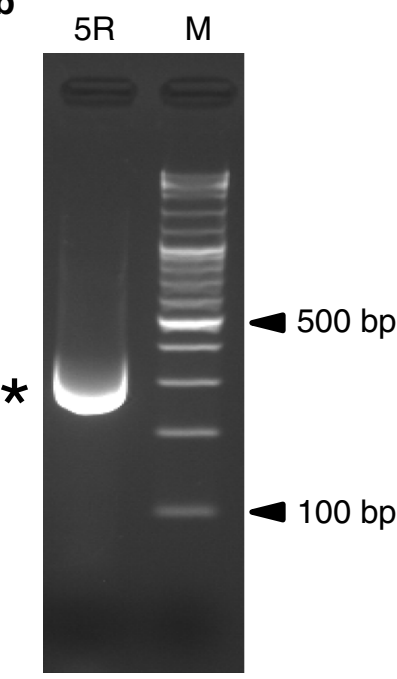

C

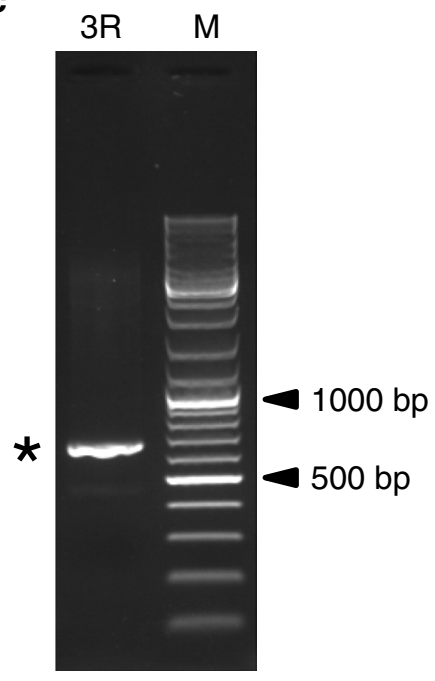

d

Tammar IGF2R

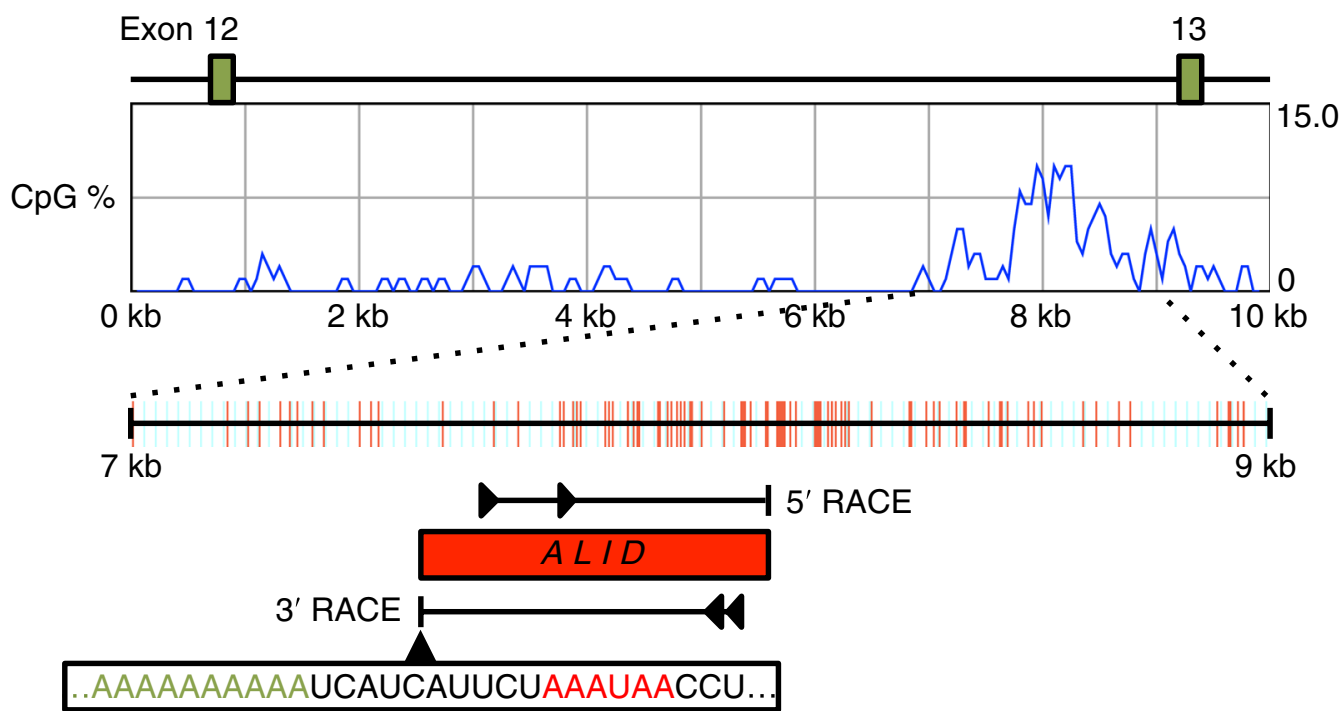

Fig. 3 Determination of the transcript structure of ALID. a Strand-specific RT-PCR to detect antisense transcript nearby tammar IGF2R DMR. BY yolk sac placenta (bilaminar region), TY yolk sac placenta (trilaminar region). b Gel photograph of 5' RACE experiment to determine transcription start site of ALID. c Gel photograph of 3' RACE experiment to determine polyadenylation site of ALID. $\mathbf{d}$ Summary illustration for the structure of ALID. The graph shows the density of CpG sites in intron 12 sequence of tammar IGF2R. Two green boxes represent position of IGF2R exon 12 and 13 in the $10 \mathrm{~kb}$ genomic sequence, respectively. The red bars indicate each $\mathrm{CpG}$ site in $2 \mathrm{~kb}$ sequence around intron $12 \mathrm{CpG}$ island. The arrowheads represent (nested) gene-specific primers used for $5^{\prime}$ and $3^{\prime}$ RACE experiments. The red box shows full-length ALID IncRNA revealed by RACE experiments. Canonical polyadenylation signal sequence (red) was found nearby polyadenylation site (green) of ALID 


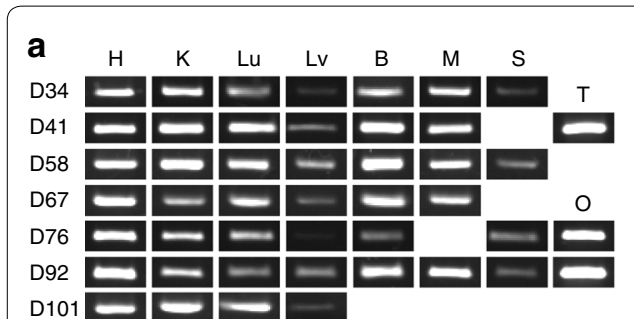

C

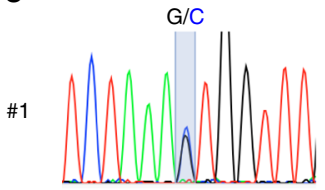
Genomic DNA

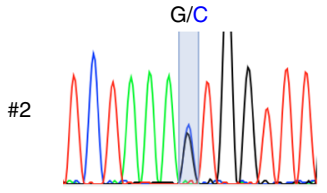

Genomic DNA

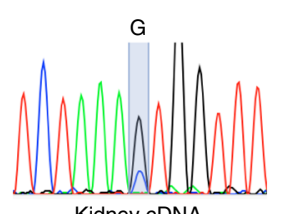
Kidney cDNA

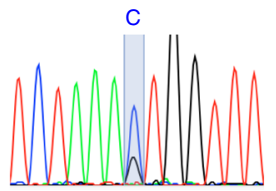

Kidney cDNA
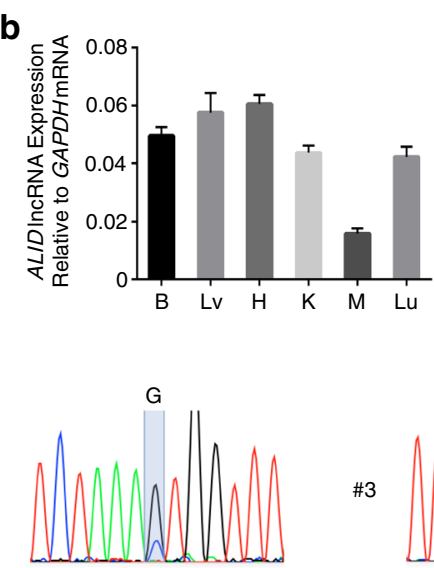

Brain CDNA

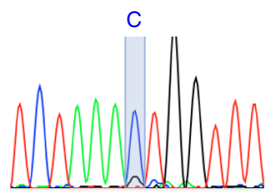

Lung cDNA

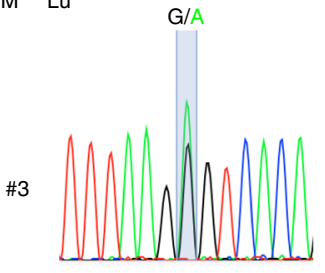

Genomic DNA

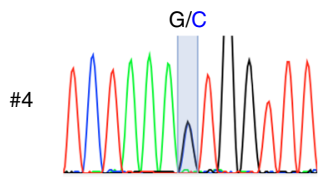

Genomic DNA

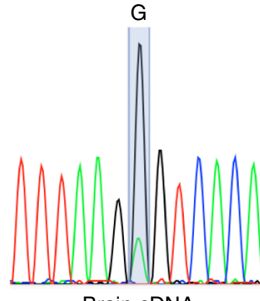

Brain cDNA

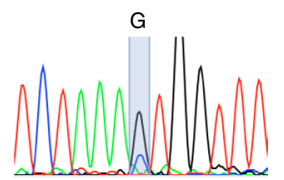

Brain cDNA

Fig. 4 Expression analyses of ALID. a Expression analysis of ALID by strand-specific RT-PCR. The left labels indicate stages of pouch young animals (days from postpartum). $H$ heart, K kidney, Lu lung, Lv liver, B brain, M skeletal muscle, $S$ spleen, $T$ testis, $O$ ovary. b Quantification of expression level of ALID by strand-specific RT-qPCR. c Allelic expression analysis of ALID by direct sequencing followed by strand-specific RT-PCR. DNA methylation analysis of nearby genomic region including these SNP sites showed that the expressed alleles were unmethylated alleles (data not shown), demonstrating paternal expression of ALID IncRNA

not shown), demonstrating the paternal expression of ALID lncRNA.

\section{Discussion}

In this study, we identified a novel antisense lncRNA, $A L I D$, analysing the IGF2R locus in an Australian marsupial, the tammar wallaby and compared it to that of opossum. We first clarified that both maternal expression and DMR of IGF2R were conserved between tammar and opossum. Confirming the evidence of the downstream DMR in IGF2R of an Australian marsupial is important to ensure the previous finding in opossum because the data of differential methylation was provided from only a single sample in the opossum study. According to the report by Das et al., the opossum IGF2R DMR was located in intron 11 [40]. However, the two exons neighbouring the tammar IGF2R DMR were exon 12 and 13 that are orthologous to exon 12 and 13 in both mouse and human $I G F 2 R$. Because all the orthologous exons of the main transcript of mouse and human IGF2R are found in both tammar and opossum genomic sequences and the tammar and opossum IGF2R DMRs were clearly located to the orthologous position, we conclude that the marsupial IGF2R DMR is present in intron 12 not 11.

While the downstream CGI in tammar IGF2R intron 12 was maternally methylated, the promoter CGI was entirely unmethylated despite maternal expression of tammar IGF2R. This observation provided good agreement with the previous work in opossum [40], suggesting that other differential epigenetic modification than DNA methylation such as differential histone marks on the $I G F 2 R$ promoter region is responsible for the paternal silencing of marsupial IGF2R. In our allelic expression analyses, there is some level of leaky expression from the repressed paternal alleles detected in all the samples examined. This might be the result of repression of the paternal tammar IGF2R promoter by DNA methylationindependent epigenetic mechanism because similar leaky expression from the repressed allele was also observed in some imprinted genes in both marsupials and eutherians that are regulated by differential histone modifications without DNA methylation $[9,11,41]$.

We also identified an antisense lncRNA transcribed from the middle of the tammar IGF2R DMR which is located in intron 12 and named it ALID. ALID was predominantly expressed from unmethylated alleles, indicating its paternal expression. Thus, despite the non-orthologous location of DMRs between mouse and tammar, Airn and ALID share a common feature that both are paternally expressed from the middle of DMRs. However, surprisingly, the length of $A L I D$ was extremely short $\sim 650 \mathrm{bp}$, compared to Airn length which is more 
than $100 \mathrm{~kb}$ [26]. This result is consistent with the strandspecific RT-PCR experiments performed in the previous study in opossum which did not detect any antisense transcript, because the position of all the primers used in their strand-specific RT-PCR experiments was too far from the DMR to detect this short transcript [40]. We fortunately detected ALID in this study because the primer position was designed closer to the middle of DMR where we presumed that the transcription start site exists. The short length of ALID suggests certain critical differences exist in the regulatory mechanisms of IGF2R imprinting between mouse and tammar. Murine Airn transcriptionally overlaps with the Igf $2 r$ promoter and this transcriptional overlap, but not the lncRNA product itself, is crucial for paternal $I g f 2 r$ silencing [27], while tammar ALID does not overlap with the IGF2R promoter due to its short length and the much more downstream intron 12 location of DMR, suggesting the marsupial IGF2R imprinting mechanism does not require the promoter overlap unlike the murine case. While the promoter overlap with Airn is critical for Igf2r imprinting, the two adjacent genes $S l c 22 a 2$ and Slc22a3 that are imprinted only in the placenta do not require promoter overlap with Airn [42]. It is thus possible that the mechanism regulating marsupial $I G F 2 R$ imprinting is similar to the promoter overlap-independent silencing mechanism regulating imprinting of Slc22a2 and Slc22a3 in the mouse placenta. Alternatively, there is a possibility that an as yet unknown very long form of $A L I D$ is expressed only in a certain developmental window in early embryonic stages and makes transcriptional overlap with IGF2R promoter. After the establishment of epigenetic silencing of paternal IGF2R, the structure of ALID may be changed to the short form, which would be functionless in this case.

Interestingly, we found that the length of the $I G F 2 R$ intron 12 has been largely extended specifically in marsupial species (Fig. 5a), while no such clear difference was observed for the intron 2 lengths among multiple species. This phenomenon is most likely linked to the marsupial-specific acquisition of the intron 12 DMR. Hence, it is simple to hypothesise that the intron 12 DMR emerged in the common ancestor of marsupials rather than that it has occurred in the common therian ancestor and only eutherians have lost it after the divergence of marsupials. Then, was the emergence of the intron 12 DMR de novo acquisition of imprinting to IGF2R or not? This question is important to discuss if the IGF2R locus had two independent origins of imprinting or just the once. We provide two simple models for the evolution of $I G F 2 R$ imprinting as it is difficult to conclude the most likely hypothesis currently (Fig. 5b). The first possibility is based on the idea that the origin of imprinting of the
$I G F 2 R$ locus occurred just once in the common therian ancestor while the change of DMR location to intron 12 and consequent loss of former DMR in intron 2 subsequently occurred in the marsupial ancestor (Fig. 5b left). Alternatively, the acquisition of IGF2R imprinting might have independently arisen separately in both the eutherian and marsupial ancestors (Fig. 5b right).

\section{Conclusions}

In this study, we provide the most likely lncRNA candidate factor for establishing paternal silencing of marsupial $I G F 2 R$ without transcriptional overlap, which is distinct from the Igf2r silencing mechanism of Airn, but may be analogous to the mode of action for the flanking Slc22a2 and Slc22a3 silencing that occurs in the mouse placenta. Although we clarified that a novel DMR in intron 12 emerged in the common ancestor of marsupials, it is still unclear whether this event was due to a change of DMR location or de novo acquisition of $I G F 2 R$ imprinting. Both scenarios represent the extraordinary history of this imprinted locus. The former scenario would provide the first evidence of the change of DMR location in any imprinted domains, and the latter scenario would be the first example of two independent origins of genomic imprinting for the same gene, suggesting extremely strong positive selection for IGF2R imprinting during mammalian evolution.

\section{Methods}

\section{Animals and tissue collection}

Tammar wallabies of Kangaroo Island origin were maintained in our breeding colony in grassy, outdoor enclosures. Lucerne cubes, grass and water were provided ad libitum and supplemented with fresh vegetables. Yolk sac placenta tissues were collected between days 21 and 26 of the 26.5 days gestation as previously described [43, 44]. Pouch young between days 34 and 101 postpartum were dissected to obtain a range of tissues. Experimental procedures conformed to Australian National Health and Medical Research Council (2013) guidelines and were approved by the Animal Experimentation Ethics Committees of the University of Melbourne.

\section{RFLP analysis}

The 3' UTR of IGF2R, including the polymorphism on the CviAII recognition sequence, was amplified by 35 cycles of RT-PCR. The PCR products were digested with 1-5 units of CviAII for $3 \mathrm{~h}$ at $25^{\circ} \mathrm{C}$. The digested PCR products were resolved by gel electrophoresis. The intensity of the cut and uncut bands was quantified with ATTO CS 


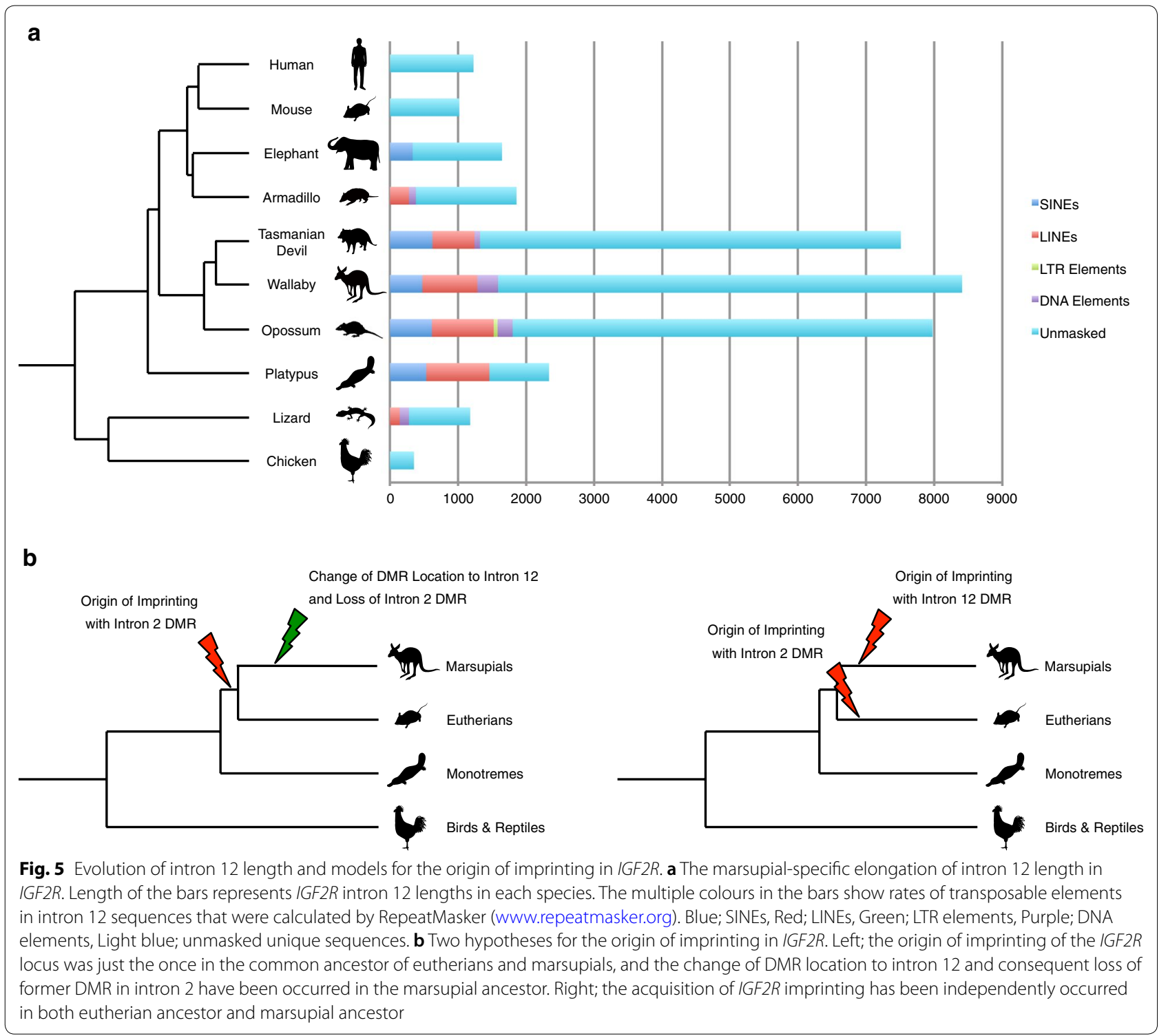

Analyzer 3 software (ATTO). Primer sequences used in this study are provided in the Additional file 1.

\section{Bisulphite sequencing}

Genomic DNA was extracted from yolk sac placentas using Trizol (Life Technologies). Genomic DNA was treated with sodium bisulphite solution, as described previously $[45,46]$. After the bisulphite treatment of the genomic DNA, 35 cycles of PCR were carried out using the primers designed by MethPrimer [47]. The PCR products were cloned using a pTAC-2 vector (BioDynamics Laboratory) and ECOS-competent Escherichia coli DH5 $\alpha$ (NIPPON GENE). Plasmids were purified using FastGene Plasmid Mini (NIPPON Genetics) and sequenced. The sequence data were analysed by QUMA (quantification tool for methylation analysis; http//quma. cdb.riken.jp) [48]. Primer sequences used in this study are provided in the Additional file 1.

\section{Strand-specific RT-PCR}

Total RNA was extracted from yolk sac placentas and a panel of pouch young tissues using Trizol (Life Technologies) as instructed by the manufacturer. Total RNA was treated with DNase I to remove genomic DNA (Promega, M6101). cDNA was synthesised using a Transcriptor First Strand cDNA Synthesis Kit (Roche) with the primer designed in the intron 12 DMR in sense direction for IGF2R. There was no amplification from the minus 
RT controls detected for any of the RNA samples examined in this study. Thirty-five cycles of PCR amplification were carried out in $10 \mu \mathrm{l}$ total volume with $10 \mathrm{ng} \mathrm{cDNA}$ using $0.2 \mathrm{U}$ TaKaRa Ex Taq HS (TaKaRa), 4 pmol of each primer and $2 \mathrm{nmol}$ of each dNTP mixture under the following cycle condition: $96^{\circ} \mathrm{C}$ for $15 \mathrm{~s}, 60^{\circ} \mathrm{C}$ for $30 \mathrm{~s}$ and $72{ }^{\circ} \mathrm{C}$ for $30 \mathrm{~s}$. PCR products were resolved by gel electrophoreses. Primer sequences used in this study are provided in the Additional file 1.

\section{$5^{\prime}$ and $3^{\prime}$ RACE experiments}

To determine the complete structure of $A L I D$, we performed $5^{\prime}$ and $3^{\prime}$ RACE experiments using a SMARTer RACE $5^{\prime} / 3^{\prime}$ Kit (Clontech) according to the manufacturer's instructions. The RACE products amplified from the templates created using pouch young brain RNA were cloned using a pTAC-2 vector (BioDynamics Laboratory) and ECOS-competent Escherichia coli DH5 $\alpha$ (NIPPON GENE). Plasmids were purified using FastGene Plasmid Mini (NIPPON Genetics) and sequenced. Primer sequences used in this study are provided in the Additional file 1 .

\section{Quantitative RT-PCR}

CDNA was prepared as described in the strand-specific RT-PCR section. Quantitative real-time polymerase chain reaction (qPCR) was carried out in triplicate in $10 \mu \mathrm{l}$ volumes containing $25 \mathrm{ng}$ cDNA, $5 \mathrm{nM}$ of the primers and FastStart Essential DNA Green Master (Roche) using LightCycler 96 (Roche). The amplification efficiency was calculated from the standard curve. GAPDH was used as the reference gene, and the data were analysed by Microsoft Excel. Primer sequences used in this study are provided in the Additional file 1.

\section{Additional file}

Additional file 1. List of primer sequences.

\section{Authors' contributions}

SS performed the experiments and analyses and was a major contributor in writing the manuscript. MBR and GS collected the tissues and contributed to the writing and editing of the manuscript. All authors contributed to the study design. All authors read and approved the final manuscript.

\section{Author details}

${ }^{1}$ Department of Agricultural and Life Sciences, Faculty of Agriculture, Shinshu University, Nagano 399-4598, Japan. ${ }^{2}$ Department of Interdisciplinary Genome Sciences and Cell Metabolism, Institute for Biomedical Sciences, ICCER, Shinshu University, Nagano 399-4598, Japan. ${ }^{3}$ School of BioSciences, The University of Melbourne, Victoria 3010, Australia.

\section{Acknowledgements}

We thank Yasuko Hayashi for technical assistance.

\section{Competing interests}

The authors declare that they have no competing interests.

Availability of data and materials

Data sharing is not applicable to this article as no datasets were generated or analysed during the current study.

\section{Consent for publication \\ Not applicable.}

\section{Ethics approval and consent to participate}

Experimental procedures conformed to Australian National Health and Medical Research Council (2013) guidelines and were approved by the Animal

Experimentation Ethics Committees of the University of Melbourne.

\section{Funding}

This work was supported by the Ministry of Education, Culture, Sports, Science and Technology (MEXT) in Japan, Program to Disseminate Tenure Tracking System (individual selection type) to SS and Australian Research Council (ARC) Discovery Project to MBR, GS and SS.

\section{Publisher's Note}

Springer Nature remains neutral with regard to jurisdictional claims in published maps and institutional affiliations.

Received: 26 June 2018 Accepted: 25 September 2018 Published online: 29 September 2018

References

1. Reik W, Lewis A. Co-evolution of X-chromosome inactivation and imprinting in mammals. Nat Rev Genet. 2005;6(5):403-10.

2. Hore TA, Rapkins RW, Graves JA. Construction and evolution of imprinted loci in mammals. Trends Genet. 2007:23(9):440-8.

3. Renfree MB, Ager El, Shaw G, Pask AJ. Genomic imprinting in marsupial placentation. Reproduction. 2008;136(5):523-31.

4. Renfree MB, Hore TA, Shaw G, Graves JA, Pask AJ. Evolution of genomic imprinting: insights from marsupials and monotremes. Annu Rev Genom Hum Genet. 2009;10:241-62.

5. Renfree MB, Suzuki S, Kaneko-Ishino T. The origin and evolution of genomic imprinting and viviparity in mammals. Philos Trans R Soc Lond B Biol Sci. 2013;368(1609):20120151.

6. Suzuki S, Shaw G, Renfree MB. Postnatal epigenetic reprogramming in the germline of a marsupial, the tammar wallaby. Epigenetics Chromatin. 2013;6(1):14

7. Pask AJ, Papenfuss AT, Ager EI, McColl KA, Speed TP, Renfree MB. Analysis of the platypus genome suggests a transposon origin for mammalian imprinting. Genome Biol. 2009;10(1):R1.

8. O'Neill MJ, Ingram RS, Vrana PB, Tilghman SM. Allelic expression of IGF2 in marsupials and birds. Dev Genes Evol. 2000;210(1):18-20.

9. Suzuki S, Renfree MB, Pask AJ, Shaw G, Kobayashi S, Kohda T, KanekoIshino T, Ishino F. Genomic imprinting of IGF2, p57(KIP2) and PEG1/MEST in a marsupial, the tammar wallaby. Mech Dev. 2005;122(2):213-22.

10. Suzuki S, Ono R, Narita T, Pask AJ, Shaw G, Wang C, Kohda T, Alsop AE, Marshall Graves JA, Kohara Y, et al. Retrotransposon silencing by DNA methylation can drive mammalian genomic imprinting. PLoS Genet. 2007;3(4):e55.

11. Ager E, Suzuki S, Pask A, Shaw G, Ishino F, Renfree MB. Insulin is imprinted in the placenta of the marsupial, Macropus eugenii. Dev Biol. 2007:309(2):317-28.

12. Smits G, Mungall AJ, Griffiths-Jones S, Smith P, Beury D, Matthews L, Rogers J, Pask AJ, Shaw G, VandeBerg JL, et al. Conservation of the H19 noncoding RNA and H19-IGF2 imprinting mechanism in therians. Nat Genet. 2008:40(8):971-6. 
13. Suzuki S, Shaw G, Kaneko-Ishino T, Ishino F, Renfree MB. Characterisation of marsupial PHLDA2 reveals eutherian specific acquisition of imprinting. BMC Evol Biol. 2011:11:244.

14. Suzuki S, Shaw G, Kaneko-Ishino T, Ishino F, Renfree MB. The evolution of mammalian genomic imprinting was accompanied by the acquisition of novel CpG islands. Genome Biol Evol. 2011;3:1276-83.

15. Stringer JM, Suzuki S, Pask AJ, Shaw G, Renfree MB. GRB10 imprinting is eutherian mammal specific. Mol Biol Evol. 2012;29(12):3711-9.

16. Stringer JM, Suzuki S, Pask AJ, Shaw G, Renfree MB. Promoterspecific expression and imprint status of marsupial IGF2. PLOS ONE. 2012;7(7):e41690.

17. Stringer JM, Suzuki S, Pask AJ, Shaw G, Renfree MB. Selected imprinting of INS in the marsupial. Epigenetics Chromatin. 2012;5(1):14

18. Kaneko-Ishino T, Kohda T, Ishino F. The regulation and biological significance of genomic imprinting in mammals. J Biochem. 2003;133(6):699-711.

19. Barlow DP, Stöger R, Herrmann BG, Saito K, Schweifer N. The mouse insulin-like growth factor type-2 receptor is imprinted and closely linked to the Tme locus. Nature. 1991;349(6304):84-7.

20. Stöger R, Kubicka P, Liu CG, Kafri T, Razin A, Cedar H, Barlow DP. Maternalspecific methylation of the imprinted mouse lgf2 $r$ locus identifies the expressed locus as carrying the imprinting signal. Cell. 1993;73(1):61-71.

21. Sleutels F, Zwart R, Barlow DP. The non-coding Air RNA is required for silencing autosomal imprinted genes. Nature. 2002:415(6873):810-3.

22. Braidotti G, Baubec T, Pauler F, Seidl C, Smrzka O, Stricker S, Yotova I, Barlow DP. The Air noncoding RNA: an imprinted cis-silencing transcript. Cold Spring Harb Symp Quant Biol. 2004;69:55-66.

23. Santoro F, Mayer D, Klement RM, Warczok KE, Stukalov A, Barlow DP, Pauler FM. Imprinted Igf2r silencing depends on continuous Airn IncRNA expression and is not restricted to a developmental window. Development. 2013:140(6):1184-95.

24. Barlow DP, Bartolomei MS. Genomic imprinting in mammals. Cold Spring Harb Perspect Biol. 2014;6(2):a018382.

25. Wutz A, Smrzka OW, Schweifer N, Schellander K, Wagner EF, Barlow DP. Imprinted expression of the lgf2r gene depends on an intronic CpG island. Nature. 1997;389(6652):745-9.

26. Lyle R, Watanabe D, te Vruchte D, Lerchner W, Smrzka OW, Wutz A, Schageman J, Hahner L, Davies C, Barlow DP. The imprinted antisense RNA at the Igf2r locus overlaps but does not imprint Mas1. Nat Genet. 2000;25(1):19-21.

27. Latos PA, Pauler FM, Koerner MV, Şenergin HB, Hudson QJ, Stocsits RR, Allhoff W, Stricker SH, Klement RM, Warczok KE, et al. Airn transcriptiona overlap, but not its IncRNA products, induces imprinted Igf2r silencing. Science. 2012;338(6113):1469-72

28. Nagano T, Mitchell JA, Sanz LA, Pauler FM, Ferguson-Smith AC, Feil R, Fraser P. The Air noncoding RNA epigenetically silences transcription by targeting G9a to chromatin. Science. 2008;322(5908):1717-20.

29. Kalscheuer VM, Mariman EC, Schepens MT, Rehder H, Ropers HH. The insulin-like growth factor type-2 receptor gene is imprinted in the mouse but not in humans. Nat Genet. 1993;5(1):74-8.

30. Ogawa O, McNoe LA, Eccles MR, Morison IM, Reeve AE. Human insulinlike growth factor type I and type II receptors are not imprinted. Hum Mol Genet. 1993;2(12):2163-5.

31. Xu Y, Goodyer CG, Deal C, Polychronakos C. Functional polymorphism in the parental imprinting of the human IGF2R gene. Biochem Biophys Res Commun. 1993;197(2):747-54.
32. Smrzka OW, Faé I, Stöger R, Kurzbauer R, Fischer GF, Henn T, Weith A, Barlow DP. Conservation of a maternal-specific methylation signal at the human IGF2R locus. Hum Mol Genet. 1995:4(10):1945-52.

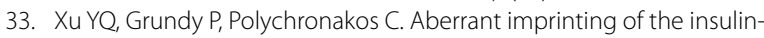
like growth factor II receptor gene in Wilms' tumor. Oncogene. 1997;14(9):1041-6.

34. Oudejans CB, Westerman B, Wouters D, Gooyer S, Leegwater PA, van Wijk IJ, Sleutels F. Allelic IGF2R repression does not correlate with expression of antisense RNA in human extraembryonic tissues. Genomics. 2001;73(3):331-7

35. Yotova IY, Vlatkovic IM, Pauler FM, Warczok KE, Ambros PF, Oshimura M, Theussl HC, Gessler M, Wagner EF, Barlow DP. Identification of the human homolog of the imprinted mouse Air non-coding RNA. Genomics. 2008:92(6):464-73.

36. O'Sullivan FM, Murphy SK, Simel LR, McCann A, Callanan JJ, Nolan CM. Imprinted expression of the canine IGF2R, in the absence of an anti-sense transcript or promoter methylation. Evol Dev. 2007;9(6):579-89.

37. Thurston A, Taylor J, Gardner J, Sinclair KD, Young LE. Monoallelic expression of nine imprinted genes in the sheep embryo occurs after the blastocyst stage. Reproduction. 2008;135(1):29-40.

38. Braunschweig MH. Biallelic transcription of the porcine IGF2R gene. Gene 2012;500(2):181-5

39. Killian JK, Byrd JC, Jirtle JV, Munday BL, Stoskopf MK, MacDonald RG, Jirtle RL. M6P/IGF2R imprinting evolution in mammals. Mol Cell. 2000:5(4):707-16.

40. Das R, Anderson N, Koran MI, Weidman JR, Mikkelsen TS, Kamal M, Murphy SK, Linblad-Toh K, Greally JM, Jirtle RL. Convergent and divergent evolution of genomic imprinting in the marsupial Monodelphis domestica. BMC Genom. 2012:13:394.

41. Lewis A, Mitsuya K, Umlauf D, Smith P, Dean W, Walter J, Higgins M, Feil $\mathrm{R}$, Reik W. Imprinting on distal chromosome 7 in the placenta involves repressive histone methylation independent of DNA methylation. Nat Genet. 2004;36(12):1291-5.

42. Sleutels F, Tjon G, Ludwig T, Barlow DP. Imprinted silencing of SIc22a2 and Slc22a3 does not need transcriptional overlap between lgf2r and Air. EMBO J. 2003:22(14):3696-704.

43. Tyndale-Biscoe CH, Renfree MB. Monographs on marsupial biology: reproductive physiology of marsupials. Cambridge: Cambridge University Press; 1987

44. Renfree MB. Review: marsupials_-placental mammals with a difference Placenta. 2010;31(Suppl):S21-6.

45. Frommer M, McDonald LE, Millar DS, Collis CM, Watt F, Grigg GW, Molloy $\mathrm{PL}$, Paul CL. A genomic sequencing protocol that yields a positive display of 5-methylcytosine residues in individual DNA strands. Proc Natl Acad Sci USA. 1992;89(5):1827-31.

46. Raizis AM, Schmitt F, Jost JP. A bisulfite method of 5-methylcytosine mapping that minimizes template degradation. Anal Biochem. 1995;226(1):161-6.

47. Li LC, Dahiya R. MethPrimer: designing primers for methylation PCRs. Bioinformatics. 2002;18(11):1427-31

48. Kumaki Y, Oda M, Okano M. QUMA: quantification tool for methylation analysis. Nucleic Acids Res. 2008;36:W170-5.

Ready to submit your research? Choose BMC and benefit from

- fast, convenient online submission

- thorough peer review by experienced researchers in your field

- rapid publication on acceptance

- support for research data, including large and complex data types

- gold Open Access which fosters wider collaboration and increased citations

- maximum visibility for your research: over 100M website views per year

At BMC, research is always in progress.

Learn more biomedcentral.com/submissions 